\title{
Nitrate Stabilizes the Rhizospheric Fungal Community to Suppress Fusarium Wilt Disease in Cucumber
}

\author{
Zechen Gu, ${ }^{1}$ Min Wang, ${ }^{1+}$ Ying Wang, ${ }^{1}$ Linxing Zhu, ${ }^{1}$ Luis Alejandro Jose Mur, ${ }^{2}$ Jun Hu, ${ }^{1}$ and \\ Shiwei Guo ${ }^{1,+}$ \\ ${ }^{1}$ Jiangsu Provincial Key Lab of Solid Organic Waste Utilization, Jiangsu Collaborative Innovation Center of Solid Organic \\ Wastes, Educational Ministry Engineering Center of Resource-saving fertilizers, Nanjing Agricultural University, Nanjing, \\ Jiangsu, China \\ ${ }^{2}$ Institute of Biological, Environmental and Rural Sciences, Aberystwyth University, Aberystwyth SY23 3DA, U.K.
}

Accepted 7 January 2020.

\begin{abstract}
Nitrogen forms can regulate soil-borne Fusarium wilt suppression, but the related mechanisms are largely unknown, especially possible action via the rhizospheric microbial community. Soil analysis, MiSeq high-throughput sequencing analysis, community diversity, and network analysis were used to characterize the impact of different nitrogen forms (nitrate and ammonium) on rhizospheric fungal communities and the contribution of nitrate to the suppression to Fusarium oxysporum f. sp. cucumerinum compared with ammonium. Nitrategrown cucumber showed a lower disease index and $F$. oxysporum f. sp. cucumerinum abundance in the rhizosphere. In comparisons with ammonium nutrients, nitrate-fed plants maintained a higher soil rhizosphere pH, microbial biomass carbon content, microbial biomass nitrogen content, as well as fungal community richness and diversity following $F$. oxysporum f. sp. cucumerinum incubation. All these factors were negatively related with disease index. Network analysis showed fewer nodes and edges in the ammonium treatments compared with nitrate treatments. The relative abundance of Pathotroph-Saprotroph, PathotrophSaprotroph-Symbiotroph, and Saprotroph fungi explained $82 \%$ of the variability of rhizosphere $F$. oxysporum f. sp. cucumerinum abundance. In conclusion, after pathogen inoculation under nitrate nutrition, the less-affected microbial composition, community diversity, and community internal relations, which resulted from the more diverse and robust microbial population, potentially contributed to greater Fusarium wilt suppression.
\end{abstract}

Keywords: ammonium, cucumber, Fusarium wilt, nitrate, rhizospheric fungal community

${ }^{\dagger}$ Corresponding authors: M. Wang; minwang@njau.edu.cn and S. Guo; sguo@njau.edu.cn

Funding: This work was financially supported by the National Key R\&D Program (2016YFD0200300), the National Basic Research Program of China (2015CB150505), the Young Elite Scientists Sponsorship Program by CAST (2018QNRC001), the Innovative Research Team Development Plan of the Ministry of Education of China (IRT_17R56) and the Fundamental Research Funds for the Central Universities (KYT201802).

*The $\boldsymbol{e}$-Xtra logo stands for "electronic extra" and indicates that supplementary materials are published online.

The author(s) declare no conflict of interest.

๑) 2020 The American Phytopathological Society
Fusarium wilt disease, caused by the fungus Fusarium oxysporum, is one of the most serious soil-borne diseases, resulting in severe losses in agriculture production (Klein et al. 2016; Wang et al. 2015). Currently, traditional strategies are used to partially control the disease, such as crop rotation (Xiong et al. 2016), fungicide treatments (Nel et al. 2007), and growing cultivars with some level of resistance (Hwang and Ko 2004). However, these strategies are usually inefficient and, in the case of fungicides, can have negative environmental impact.

Previous studies have reported that mineral nutrients are important in improving plant disease resistance (Amtmann et al. 2008; Dordas 2008). In particular, the agricultural impact of nitrogen nutrition on disease development has been extensively examined (Huber and Watson 1974) but with different effects being noted. Increasing nitrogen supply could decrease the susceptibility of tomato to Botrytis cinerea (Hoffland et al. 2000), but excess nitrogen supply increases the incidence of rice blast (Long et al. 2000). Beyond altered nitrogen availability, the impact of nitrogen forms on disease development and plant resistance has been widely demonstrated in previous studies (Mur et al. 2017). The most widely accepted mechanisms related to increased plant susceptibility with $\mathrm{NH}_{4}^{+}$nutrition are enhanced content of apoplastic sugar, amino acids, as well as $\gamma$-amino-n-butyric acid, which would increase availability of nutrients to the pathogen (Gupta et al. 2013). Besides, the increased plant resistance with $\mathrm{NO}_{3}{ }^{-}$ nutrition is considered to be associated with the generation of nitric oxide (NO) and increased content of polyamine, a metabolite that is involved in systemic resistance, penetration defense, and signal transduction (Gupta et al. 2013; Tiburcio et al. 2014; Walters et al. 2002). For Fusarium wilt, specifically, $\mathrm{NH}_{4}^{+}$nutrition is beneficial for the activation of virulence mechanisms such as penetration, vegetative hyphal fusion, and root adhesion (López-Berges et al. 2010; Zhou et al. 2017).

Since rhizosphere is an important area in which soil-borne pathogens first interact with plant hosts, its role in regulating disease development has been examined in several plant pathogens. Pathogens need to compete with the rhizosphere microbiome for available nutrients and microsites as the result of rhizodeposition (Chapelle et al. 2016), which is an important exchange process between plant/soil metabolites (Hartmann et al. 2008). For example, the production of secondary metabolites that suppress pathogen growth (Haas and Défago 2005) can increase with the density and richness of the inoculated probiotic consortia (Jousset et al. 2014). Therefore, the 
biodiversity of the introduced rhizosphere communities could affect competition with other microorganisms, including both the resident microbiota and pathogens (Wei et al. 2015). Further, the microbial community associated with plant roots has been shown to be essential for the suppression of soil-borne pathogens (Xiong et al. 2017) and multispecies probiotica may enhance the disease suppression efficacy of tomato (Hu et al. 2016).

Even though the mechanisms related to disease resistance with different nitrogen forms are widely studied, the potential role of the manipulation of the rhizosphere microbial community through nitrogen forms in defensing pathogens is currently lacking. Thus, we investigated the effects of different nitrogen forms on the chemical and microbiological characteristics of the rhizosphere with a focus on how nitrogen forms could affect the incidence of cucumber Fusarium wilt disease caused by Fusarium oxysporum f. sp. cucumerinum.

\section{RESULTS}

\section{Effect of different nitrogen forms}

on the disease tolerance of cucumber.

Initial assessments of the impact of nitrogen forms on F. oxysporum f. sp. cucumerinum development focused on symptom development and imaging leaf temperature changes. As shown in Figure 1A, the symptoms of yellowing and wilting after inoculation with $F$. oxysporum f. sp. cucumerinum were observed in the bottom leaves of ammonium-grown plants but not in nitrate-grown. No significant difference in leaf temperature was observed between $\mathrm{NH}_{4}{ }^{+}$- and $\mathrm{NO}_{3}{ }^{-}$-fed (A and N, respectively) plants (Fig. 1B). Independent of nitrogen forms, leaf temperature was significantly increased after inoculation with $F$. oxysporum $\mathrm{f}$. sp. cucumerinum. In a comparison with $\mathrm{A}$ and $\mathrm{N}$ treatments, leaf temperature in treatments after inoculation with $F$. oxysporum f. sp. cucumerinum under $\mathrm{NH}_{4}{ }^{+}$and $\mathrm{NO}_{3}^{-}$(AI and NI, respectively) nutrition was increased by 17.4 and $4.68 \%$, respectively (Fig. 1B). Both F. oxysporum f. sp. cucumerinum abundance in the rhizosphere and the disease index were increased after inoculation with $F$. oxysporum f. sp. cucumerinum, and significantly greater $F$. oxysporum f. sp. cucumerinum abundance and a higher disease index were observed in AI plants compared with NI (Fig. 1C and D).

\section{Effects of different nitrogen forms on chemical and microbiological properties following inoculation with $F$. oxysporum f. sp. cucumerinum.}

Different nitrogen forms affected biological and nonbiological characteristics of the rhizosphere soil. As shown in Table 1, the rhizosphere soil was more alkaline in nitratetreated samples than the ammonium treated equivalents. The rhizosphere soil $\mathrm{pH}$ values were 6.68 in A plants and 7.08 in $\mathrm{N}$ plants after $F$. oxysporum f. sp. cucumerinum inoculation, they increased by $1.8(\mathrm{AI})$ and $1.4 \%(\mathrm{NI})$, respectively. A significant negative relationship between rhizosphere soil $\mathrm{pH}$ and disease index was obtained (Supplementary Table S1). The ammonium/nitrate ratio $(\mathrm{A} / \mathrm{N})$ was positively related with the disease index (Supplementary Table S1). No significant differences in soil available phosphorus, soil available potassium, soil total carbon, and soil total nitrogen content were observed between $\mathrm{AI}$ and NI treatments. Both soil microbial biomass carbon $(\mathrm{MBC})$ and nitrogen $(\mathrm{MBN})$ were negatively related to disease index and were decreased following inoculation with F. oxysporum f. sp. cucumerinum in plants fed $\mathrm{NH}_{4}{ }^{+}$, while there was no significant difference in MBC and MBN between $\mathrm{N}$ and NI treatments (Table 1; Supplementary Table S1).
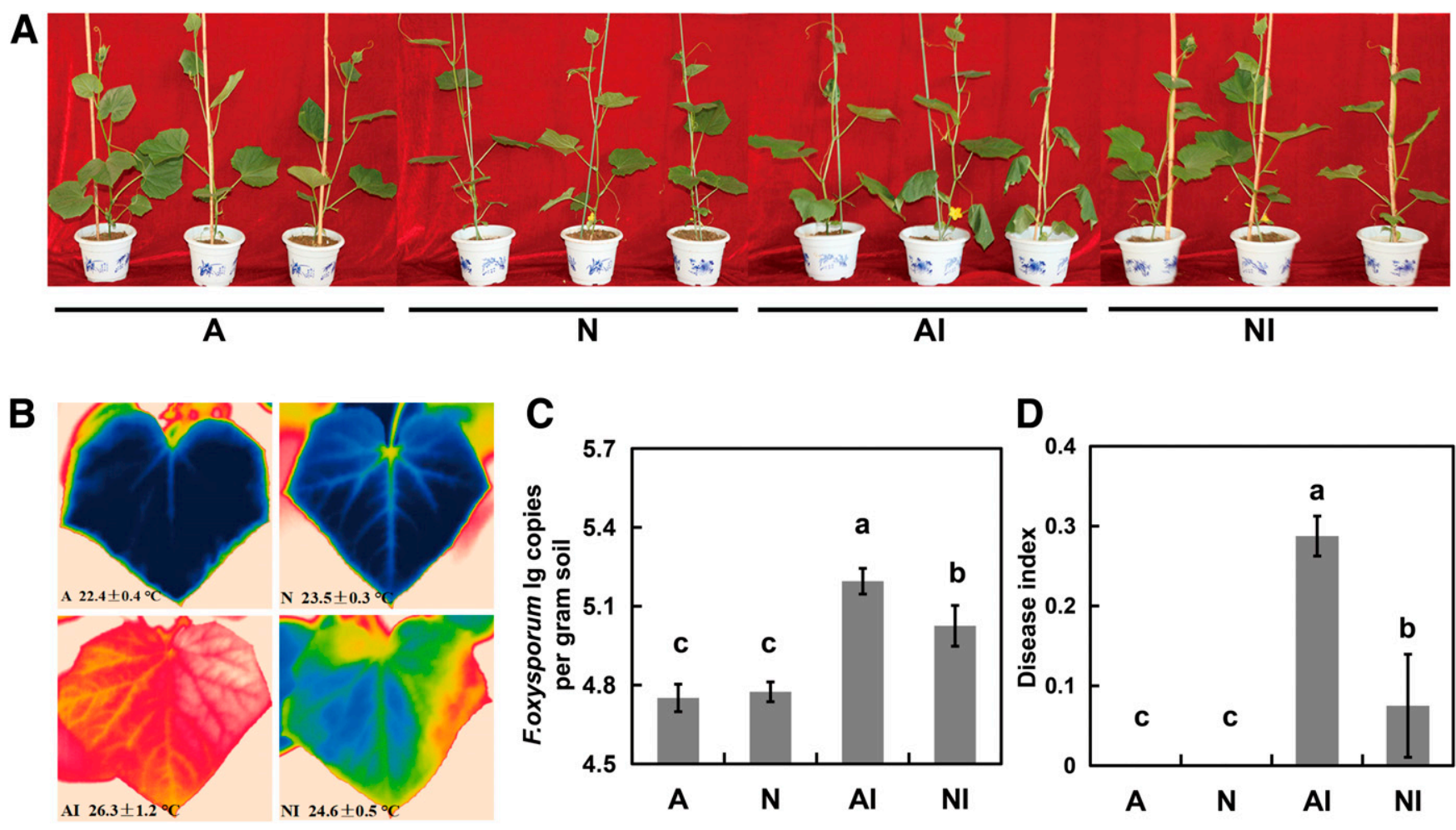

Fig. 1. Effect of different nitrogen forms on cucumber Fusarium wilt disease and the abundance of Fusarium oxysporum f. sp. cucumerinum in rhizosphere soil. A, Disease symptoms post inoculation. B, Thermal images of cucumber plants post inoculation. C, The abundance of Fusarium oxysporum (log copies per gram of dry soil) in cucumber rhizosphere soil. $\mathbf{D}$, Disease index of cucumbers in different treatments. The numbers in thermal images indicates the average temperature of the leaf blade. The disease indices reflected the proportion of diseased leaves. Bars represent the standard deviation of the four replicates, and different letters above the bars indicate a significant difference at the 0.05 probability level based on one-way analysis of variance. 
Effects of nitrogen forms on rhizosphere fungi composition and community diversity

with $F$. oxysporum f. sp. cucumerinum inoculation.

Assessments of fungal operational taxonomic units (OTUs) indicated the predominance of the Ascomycota phylum, which accounted for $82 \%$ of all those detected in rhizosphere soil (Supplementary Fig. S1a). In order of abundance, the next three phyla were Basidiomycota, Rozellomycota, Zygomycota (10, 4 , and $3 \%$, respectively). The fungal phyla in ammonium treatments were greatly affected by inoculation of $F$. oxysporum f. sp. cucumerinum and were characterized by a decrease in the relative abundance of ascomycota and increase in basidiomycota (Supplementary Fig. S1b). STAMP analysis indicated that $F$. oxysporum f. sp. cucumerinum inoculation affected the abundance of detected genera, and this phenomenon was much more significant in ammonium treatments (Fig. 2). The relative abundance of nine genera, including Penicillium, Fusarium, Bullera, Myrothecium, Apodospora, Acaulospora, Acremonium, Stachybotrys and Alternaria was markedly increased $(P<0.05)$ after $F$. oxysporum f. sp. cucumerinum inoculation with ammonium treatment, while only one genus (Apodospora) was significantly increased after inoculation in nitrate treated plants (Fig. 2).

The richness and diversity of each treatment were calculated based on the Chaol index and Shannon index of the fungal community. In order to avoid the effect of $F$. oxysporum OTUs on the responses of richness and diversity, we excluded OTUs of $F$. oxysporum from the alpha-diversity analysis. Both of Chao 1 and Shannon indexes were lower in ammonium fed plants than that in nitrate fed plants after inoculation with F. oxysporum f. sp. cucumerinum (Fig. 3). The Chao 1 and Shannon indexes were negatively correlated with disease index (Supplementary Table S1).

The beta diversity was measured by using Bray-Curtis distances and displayed using an analysis of principal coordinates, which revealed a clear differentiation of samples belonging to the A, N, AI and NI rhizosphere soil that explained $51.2 \%$ of the overall variance of the data (Fig. 4A). The first principal component (PC1) of the principal component analyses was significantly related with disease index (Supplementary Table $\mathrm{S} 1$ ). The effects of $F$. oxysporum themselves can be avoided by excluding $F$. oxysporum OTUs from the beta-diversity analysis, and we found the variance between treatments $(44.78 \%)$ and interpretation of CPCoA1 (39.17\%) were both reduced, the dispersion between points of $\mathrm{N}$ and NI is significantly reduced after excluding $F$. oxysporum OTUs (Fig. 4B).
Effect of nitrogen forms on microbial molecular ecological network of rhizosphere fungal community following

F. oxysporum f. sp. cucumerinum inoculation.

As Figure 5 showed, network analysis was conducted on different microbial communities and the topological properties of the obtained networks indicated that in general, the number of nodes and edges in A and AI treatment was lower than that in the nitrate treatments (N, NI) (Table 2). A total of 65 nodes, 396 edges, and the modularity was 0.56 with 3 modules was found in A treatment, and AI treatment possessed 51 nodes, 345 edges, and the modularity was 0.45 with 2 modules. For $\mathrm{N}$ treatment, the network had 86 nodes, 610 edges, and the modularity was 0.51 with 3 modules, and NI possessed 89 nodes, 621 edges and the modularity was 0.57 with 4 modules. As the connection density of the node in the Network can be used as a reference for evaluating the influence of the node, here we defined the nodes with the top 10 number of interlinks in each network as net hubs which were, by implication, key in mediating other species in the community. The species at each hub are listed in Supplementary Table S2. Only one hub (Otu153) was shared in A and AI treatments, while four hubs (Otu137, Otu173, Otu184 and Otu194) were common to both N and NI treatment. These results suggested that nitrate nutrition had a great contribution to a greater resilience in topologically active species in rhizosphere fungal community.

\section{Effect of different nitrogen forms on functional fungi composition in rhizosphere with $F$. oxysporum f. sp. cucumerinum inoculation.}

The correlation between the OTUs and $F$. oxysporum f. sp. cucumerinum abundance (as assessed by qPCR) were conducted in each treatment and a total of 7 trophic mode groups were collected. By using the general linear model (GLM), a model with three variables explained the maximum variation $(82 \%)$ in $F$. oxysporum f. sp. cucumerinum abundance, including Pathotroph-Saprotroph, Pathotroph-SaprotrophSymbiotroph and Saprotroph (Supplementary Table S3). Relationships between these three trophic fungi and $F$. oxysporum f. sp. cucumerinum abundance are showed in Table 3, which suggested that most of the Saprotroph fungi were negatively related with $F$. oxysporum f. sp. cucumerinum abundance. The first principal component (F-PC1) of the principal component analyses based on Pathotroph-Saprotroph, PathotrophSaprotroph-Symbiotroph and Saprotroph abundance was significantly related with disease index (Supplementary Table S1).

Table 1. Effects of nitrogen forms and Fusarium oxysporum f. sp. cucumerinum inoculation in cucumber plants ${ }^{\mathrm{Z}}$

\begin{tabular}{|c|c|c|c|c|}
\hline Effect on & $\mathbf{A}$ & $\mathbf{N}$ & AI & NI \\
\hline $\mathrm{pH}$ & $6.68 \pm 0.04 \mathrm{~d}$ & $7.04 \pm 0.03 b$ & $6.80 \pm 0.07 \mathrm{c}$ & $7.14 \pm 0.02 \mathrm{a}$ \\
\hline $\mathrm{NH}_{4}^{+}-\mathrm{N}(\mathrm{mg} / \mathrm{kg})$ & $56.9 \pm 7.6 b$ & $14.3 \pm 3.4 \mathrm{c}$ & $66.9 \pm 3.1 \mathrm{a}$ & $11.2 \pm 1.0 \mathrm{c}$ \\
\hline $\mathrm{NO}_{3}^{-}-\mathrm{N}(\mathrm{mg} / \mathrm{kg})$ & $38.7 \pm 6.8 b$ & $91.5 \pm 17.5 \mathrm{a}$ & $34.3 \pm 3.7 b$ & $97.9 \pm 13.2 \mathrm{a}$ \\
\hline $\mathrm{A} / \mathrm{N}$ & $1.5 \pm 1.1 \mathrm{~b}$ & $0.5 \pm 0.1 \mathrm{c}$ & $1.9 \pm 0.8 \mathrm{a}$ & $0.2 \pm 0.1 \mathrm{c}$ \\
\hline $\mathrm{AP}(\mathrm{mg} / \mathrm{kg})$ & $42.4 \pm 3.2 \mathrm{a}$ & $42.7 \pm 4.0 \mathrm{a}$ & $47.4 \pm 2.4 \mathrm{a}$ & $41.8 \pm 1.7 \mathrm{a}$ \\
\hline $\mathrm{AK}(\mathrm{mg} / \mathrm{kg})$ & $102 \pm 3.6 b$ & $113 \pm 5.2 \mathrm{a}$ & $98.9 \pm 7.8 b$ & $99.6 \pm 5.7 \mathrm{~b}$ \\
\hline $\mathrm{TC}(\mathrm{g} / \mathrm{kg})$ & $7.70 \pm 0.52 \mathrm{a}$ & $8.14 \pm 0.34 \mathrm{a}$ & $8.18 \pm 0.76 \mathrm{a}$ & $7.86 \pm 0.71 \mathrm{a}$ \\
\hline $\mathrm{TN}(\mathrm{g} / \mathrm{kg})$ & $0.78 \pm 0.06 \mathrm{a}$ & $0.82 \pm 0.04 \mathrm{a}$ & $0.82 \pm 0.06 \mathrm{a}$ & $0.80 \pm 0.07 \mathrm{a}$ \\
\hline $\mathrm{C} / \mathrm{N}$ & $9.85 \pm 0.18 \mathrm{a}$ & $9.98 \pm 0.28 \mathrm{a}$ & $9.98 \pm 0.28 \mathrm{a}$ & $9.78 \pm 0.15 a$ \\
\hline $\mathrm{MBC}(\mathrm{mg} / \mathrm{kg})$ & $63.3 \pm 4.5 \mathrm{ab}$ & $70.7 \pm 2.0 \mathrm{a}$ & $56.8 \pm 7.7 \mathrm{~b}$ & $70.1 \pm 4.4 \mathrm{a}$ \\
\hline $\mathrm{MBN}(\mathrm{mg} / \mathrm{kg})$ & $9.6 \pm 1.1 \mathrm{a}$ & $10.6 \pm 1.4 \mathrm{a}$ & $7.3 \pm 1.0 \mathrm{~b}$ & $10.0 \pm 0.7 \mathrm{a}$ \\
\hline Microbial C/N & $6.6 \pm 0.6 \mathrm{a}$ & $6.7 \pm 0.8 \mathrm{a}$ & $7.8 \pm 0.4 \mathrm{a}$ & $7.0 \pm 0.5 \mathrm{a}$ \\
\hline
\end{tabular}

z Plants were supplied with ammonium fertilizer (A), nitrate fertilizer (N), ammonium fertilizer and inoculated with $F$. oxysporum f. sp. cucumerinum (AI), nitrate fertilizer and inoculated with $F$. oxysporum $\mathrm{f}$. sp. cucumerinum $(\mathrm{NI}) . \mathrm{A} / \mathrm{N}=$ the ratio of $\mathrm{NH}_{4}{ }^{+}$to $\mathrm{NO}_{3}{ }^{-}$content, $\mathrm{AP}=$ available phosphorus, $\mathrm{AK}=$ available potassium, $\mathrm{TC}=$ total carbon, $\mathrm{TN}=$ soil total nitrogen, $\mathrm{C} / \mathrm{N}=$ the ratio of soil total carbon to nitrogen, $\mathrm{MBC}=$ soil microbial biomass carbon, $\mathrm{MBN}=$ soil microbial biomass nitrogen, and microbial $\mathrm{C} / \mathrm{N}=$ the ratio of soil microbial biomass carbon to nitrogen in cucumber plants. Values are showed as means \pm standard deviation. Means followed by the same letter for a given factor are not significantly different $(P<0.05)$. 


\section{DISCUSSION}

Nitrogen forms were proved to affect numerous plant disease developments, in the present study, nitrate nutrition supply decreased $F$. oxysporum f. sp. cucumerinum abundance in the rhizosphere and increased the suppression to Fusarium wilt caused by $F$. oxysporum f. sp. cucumerinum in cucumber, which was accordant with previous studies (Fig. 1) (Wang et al. 2016; Zhou et al. 2017). The soil in our experiment comes from the cucumber farmland, therefore, the selection to the microbial species by the early cucumber planting may cause the high abundance of $F$. oxysporum in the noninoculated treatments (Fig. 1C) (Huang et al. 2018), besides, in rhizosphere soil, the plant-transformed pathogenic microorganisms are generally enriched, which may be driven by plant root (Mendes et al. 2018a).

More recent research has shown that the nitrogen assimilation directly affects the carbon and nitrogen sources for the microbial biomass and thereby regulates the microbial community (Sasse et al. 2018; Xiao et al. 2018). However, the response of the microbial community in the rhizosphere soil subject to different nitrogen forms was unknown. Our present work studied Fusarium wilt in plants supplied with different nitrogen forms and focused on the possible role of rhizospheric microbial community assembly in influencing disease severity. MBC and MBN biomass are important indicators of soil microbial community activity (Hortal et al. 2013; Heuck et al. 2015 ) and, more pertinent to our study, are commonly higher in disease-suppressive soil (Liu et al. 2007a; Liu et al. 2007b). Our results showed that nitrate rather than ammonium elevated soil $\mathrm{pH}$ and maintained the soil microbial biomass carbon and nitrogen content (Table 1). Therefore, changes in MBC and MBN content caused by the utilization of nitrogen may play a key role in soil disease resistance. As evidence for this, Pearson correlation analysis showed that both $\mathrm{MBC}$ and $\mathrm{MBN}$ were
A

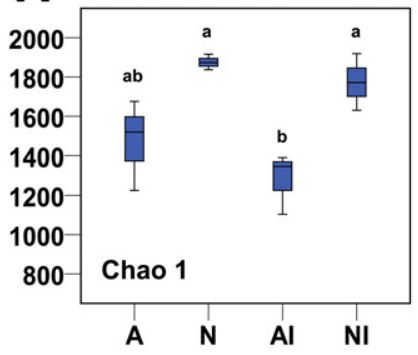

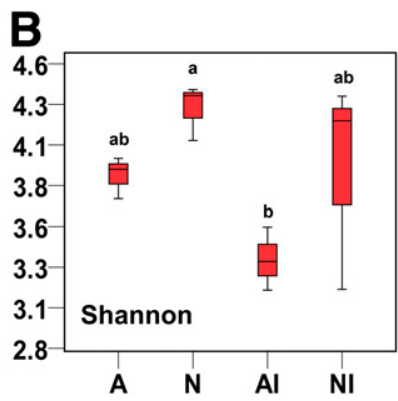

Fig. 3. Effects of different nitrogen forms and Fusarium oxysporum f. sp. cucumerinum infection on the $\mathbf{A}$, Chao 1 and $\mathbf{B}$, Shannon indices. The boxes mean the result of the operational taxonomic units excluding $F$. oxysporum. Different letters indicate a significant difference among treatments by oneway analysis of variance $(P<0.05)$. A = with ammonium fertilizer, $\mathrm{N}=$ with nitrate fertilizer, $\mathrm{AI}=$ with ammonium fertilizer and inoculated with $F$. oxysporum f. sp. cucumerinum, and $\mathrm{NI}=$ with nitrate fertilizer and inoculated with $F$. oxysporum f. sp. cucumerinum.
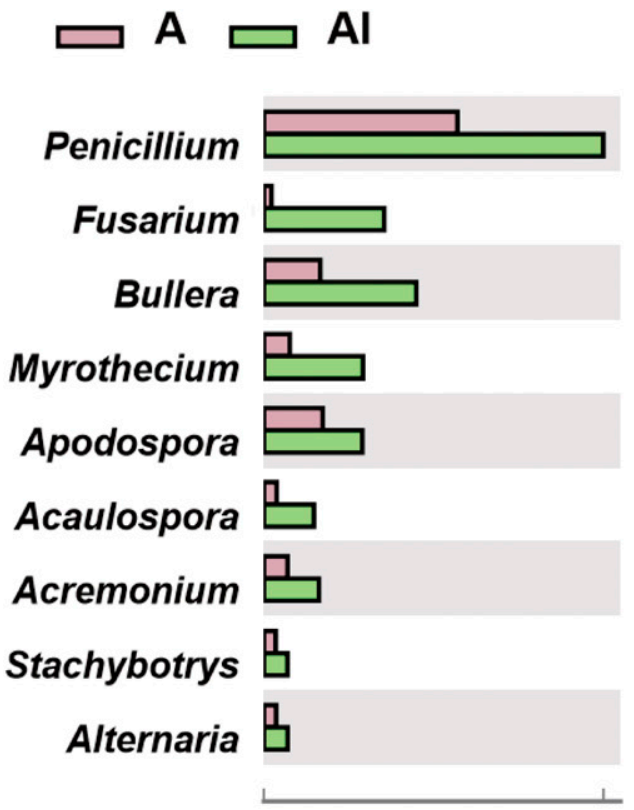

$95 \%$ confidence intervals

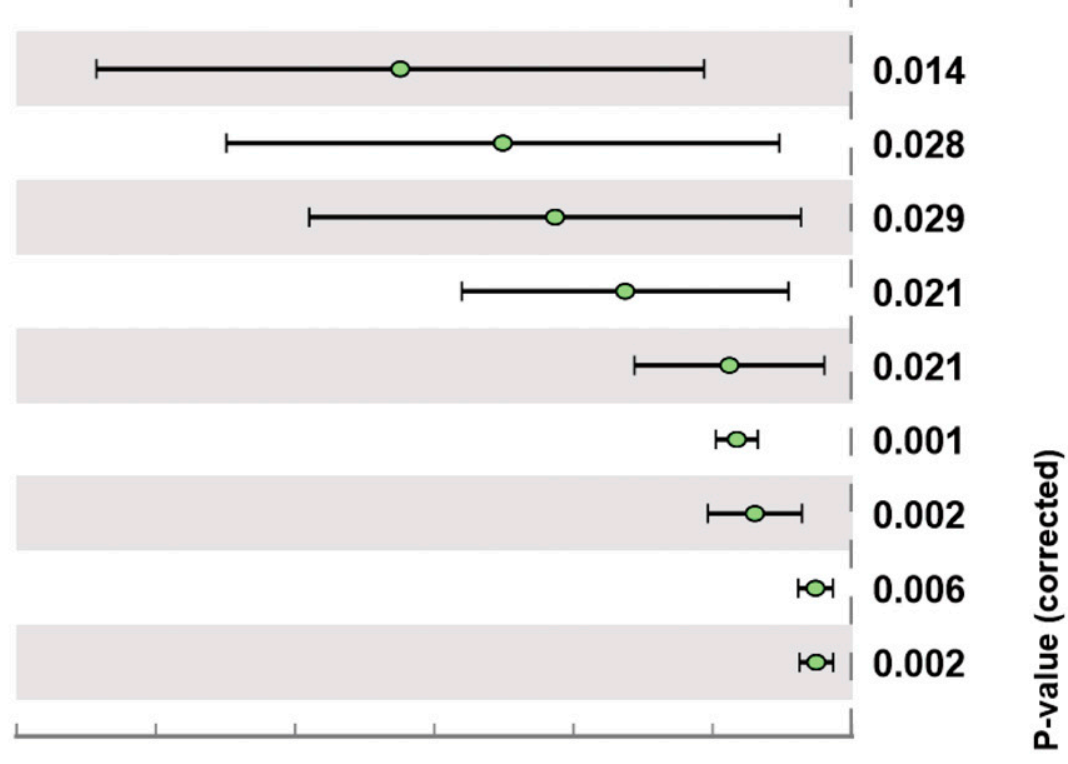

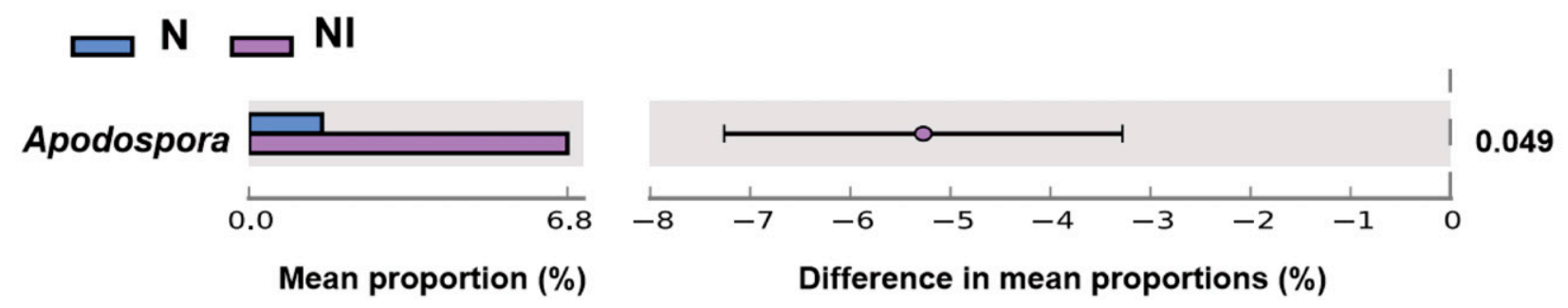

Fig. 2. Effects of Fusarium oxysporum f. sp. cucumerinum infection on the genera abundance under different nitrogen forms. Mean proportion (\%) is based on the relative abundances from next generation sequencing analysis. Only fungi with significant differences $(P<0.05)$ are listed. A $=$ with ammonium fertilizer, $\mathrm{N}=$ with nitrate fertilizer, $\mathrm{AI}=$ with ammonium fertilizer and inoculated with $F$. oxysporum $\mathrm{f}$. sp. cucumerinum, and $\mathrm{NI}=$ with nitrate fertilizer and inoculated with F. oxysporum f. sp. cucumerinum. 
negatively correlated with the disease index (Supplementary Table S1). These results suggested that the improved resistance of cucumber to $F$. oxysporum f. sp. cucumerinum with nitrate may relate to the microbial community in rhizosphere soil.

Analysis of the composition, diversity, ecological network structure, and functional characteristics of microbial communities can be used to explore the role of rhizosphere fungal community in disease resistance process (Guo et al. 2017; Mendes et al. 2018b). Our treatments with different nitrogen forms and $F$. oxysporum f. sp. cucumerinum inoculation had dramatic effects on the rhizosphere microbial communities. We found that the abundance of genera in the ammoniumtreated rhizosphere exhibited the greater response were to $F$. oxysporum f. sp. cucumerinum inoculation, as compared with nitrate. With nitrate treated soils, the rhizosphere fungal community structure was more stable than with ammonium, which could enhance the ability of rhizosphere microbial community to resist pathogen ( $F$. oxysporum f. sp. cucumerinum) invasion. In plant, $\mathrm{NO}$ signaling associated with $\mathrm{NO}_{3}{ }^{-}$supply can enhance plant resistance (Durner and Klessig 1999) and in rhizosphere nitrate may be a more suitable nitrogen form for fungi to increase stability (Celar 2003). The relationship between soil-borne diseases and soil Penicillium or Apodospora has been reported in previous studies (Soustrade et al. 2000; van Bruggen et al. 2016). The abundance of Penicillium usually increased after the $F$. oxysporum enriched in soil, while the abundance of Apodospora is negatively correlated with the incidence of Fusarium wilt (Meng et al. 2017). Penicillium is considered to be a potential pathogen in the soil after the occurrence of Fusarium wilt, and the suppression of Apodospora to disease may be related to its ability to decompose complex polysaccharides (Hakulinen et al. 2003). Combined with our results, it can be anticipated that the increase of Apodospora relative abundance in both AI and NI may be the result of the soil microbial community itself resisting the pathogen, and the increase of Penicillium relative abundance in AI cloud be due to the transformation of soil into a diseaseconducive soil.

To explore whether the increase in genus Fusarium is derived from inoculation or a side-effect of inoculation, we analyzed fungal diversity after excluding the OTUs of $F$. oxysoprum. The reductions were seen with treatments with both nitrogen forms after $F$. oxysporum f. sp. cucumerinum inoculation with Chao 1 and Shannon indices negatively correlated with disease index (Supplementary Table S1). However, in the case of nitrate, the reductions were not significantly different. Except for maintaining diversity, increased fungal abundance in the rhizosphere under nitrate nutrition would increase competition for resources in the rhizosphere, which could further reduce pathogenic population growth and contribute to plant protection against fungal infection. Previous studies on the disease-suppressive soil also showed higher richness and diversity of soil fungal community in disease-suppressive soil than that in diseaseconducive soil (Rosenzweig et al. 2012; Shen et al. 2015b). When the OTUs of $F$. oxysporum were excluded, the results of both alpha and beta diversity indicated that the variation of AI to A was significant and the difference between NI and N was reduced. It means that the effect of $F$. oxysporum f. sp. cucumerinum inoculation on AI involved other fungal species in addition to itself, while other species in NI were less affected.

Based on microbial molecular ecological network analyses, our results showed a more active species and interactions with nitrate supply compared with ammonium treatments, which indicated a higher levels of complexity under nitrate nutrition. This implied that diverse communities exhibited a higher number of species interactions and intensified competition for niche space (van Elsas et al. 2012). As a result, plant resistance to disease through rhizospheric diversity could be mechanistically explained by the underlying interaction network architecture (Wei et al. 2015). The network of the nitrate treatment inoculated with $F$. oxysporum f. sp. cucumerinum showed the highest modular structure and a lower average path length, modularity combined with a short average path length, which suggested a more prompt response by the microbial community to environmental perturbations (Faust and Raes 2012). Such a modular structure suggests diversity in the roles and functionality of the microbial species to increase niche overlap (Poudel et al. 2016). It was speculated that a highly connected and modular rhizosphere could decrease pathogen invasion due to more efficient consumption for resources by a diversity
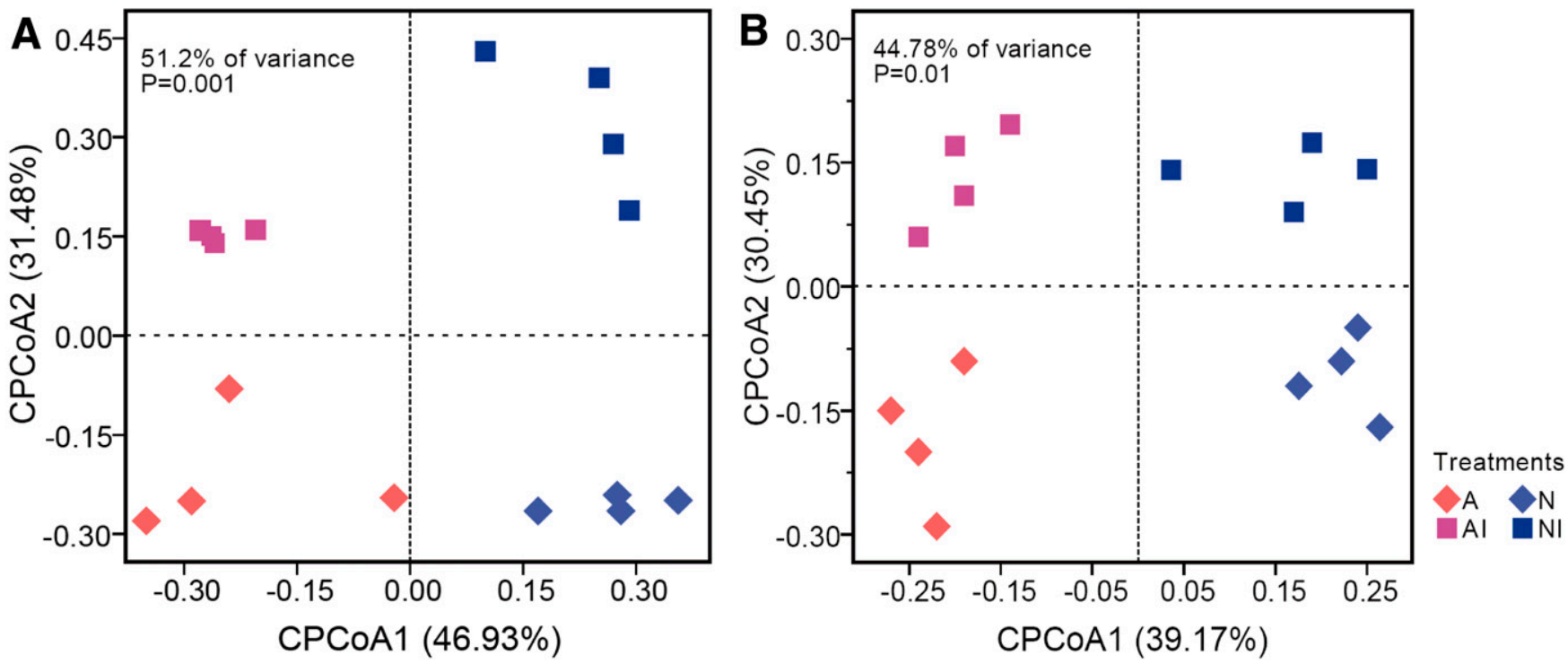

Fig. 4. Effects of different nitrogen forms and Fusarium oxysporum f. sp. cucumerinum infection on beta-diversity. A, Constrained PCoA plot of Bray-Curtis distances between samples constrained by treatments based on all operational taxonomic units (OTUs) and B, the OTUs excluding F. oxysporum. The percentage of variation indicated in each axis corresponds to the fraction of the total variance explained by the projection. $\mathrm{A}=$ with ammonium fertilizer, $\mathrm{N}=$ with nitrate fertilizer, $\mathrm{AI}=$ with ammonium fertilizer and inoculated with $F$. oxysporum $\mathrm{f}$. sp. cucumerinum, and $\mathrm{NI}=$ with nitrate fertilizer and inoculated with F. oxysporum f. sp. cucumerinum. 
microbial community (van Elsas et al. 2012; Wei et al. 2015). In network analysis, the hubs indicated the most important nodes, which may be interpreted as key taxa inside a connected community (Zhu et al. 2016), and the network hubs (assessed by the number of connections) commonly were used to indicate the centrality. After inoculation with pathogen $(F$. oxysporum $\mathrm{f}$. sp. cucumerinum), we found that the ability to maintain the stability of the ecological network of fungal communities was stronger in nitrate treatment because the active species were less likely to be displaced by $F$. oxysporum f. sp. cucumerinum inoculation (Supplementary Table S2).

The FUNGuild database allows the classification of fungal sequences into 12 functional groups based on trophic mode. This bioinformatic tool was used to understand microbial function in rhizosphere samples. We analyzed the relative abundance of Pathotroph-Saprotroph, Pathotroph-SaprotrophSymbiotroph and Saprotroph fungi and the optimal GLM model explained $82 \%$ of the variability of rhizosphere $F$. oxysporum f. sp. cucumerinum abundance. A large number of negative correlations were found in Saprotroph fungi (Table 3), which indicated that many rhizospheric Saprotrophic fungi may inhibit $F$. oxysporum f. sp. cucumerinum growth. It is obvious that a pathogen is required to occupy a rhizospheric niche for at least a short period in order to infect the host (Mallon et al. 2018). Therefore, the ability of rhizosphere indigenous microbes to compete for space and resources could determine whether the pathogen can successfully seize the niche (Bödeker et al. 2016).

In conclusion, our results suggested that different nitrogen forms results in different rhizosphere fungal community structure and function, both of which were contributed to the increased resistance of nitrate nutrition to Fusarium wilt disease. The possible resistant mechanisms are summarized in Figure 6. In our model, we proposed that nitrate is particularly effective in enhancing the functional resilience of rhizosphere fungal community following inoculation of $F$. oxysporum $\mathrm{f}$. sp. cucumerinum by maintaining a higher rhizosphere $\mathrm{pH}, \mathrm{MBC}$ and MBN, as well as a more diverse and stable fungal community. The robust microbial population with few changes following $F$. oxysporum f. sp. cucumerinum incubation under nitrate nutrition ensured niche occupancy and the community function (F-PC1) of this rhizosphere, by which the successful competition for nutrients enhanced the resistance of cucumber to Fusarium wilt.

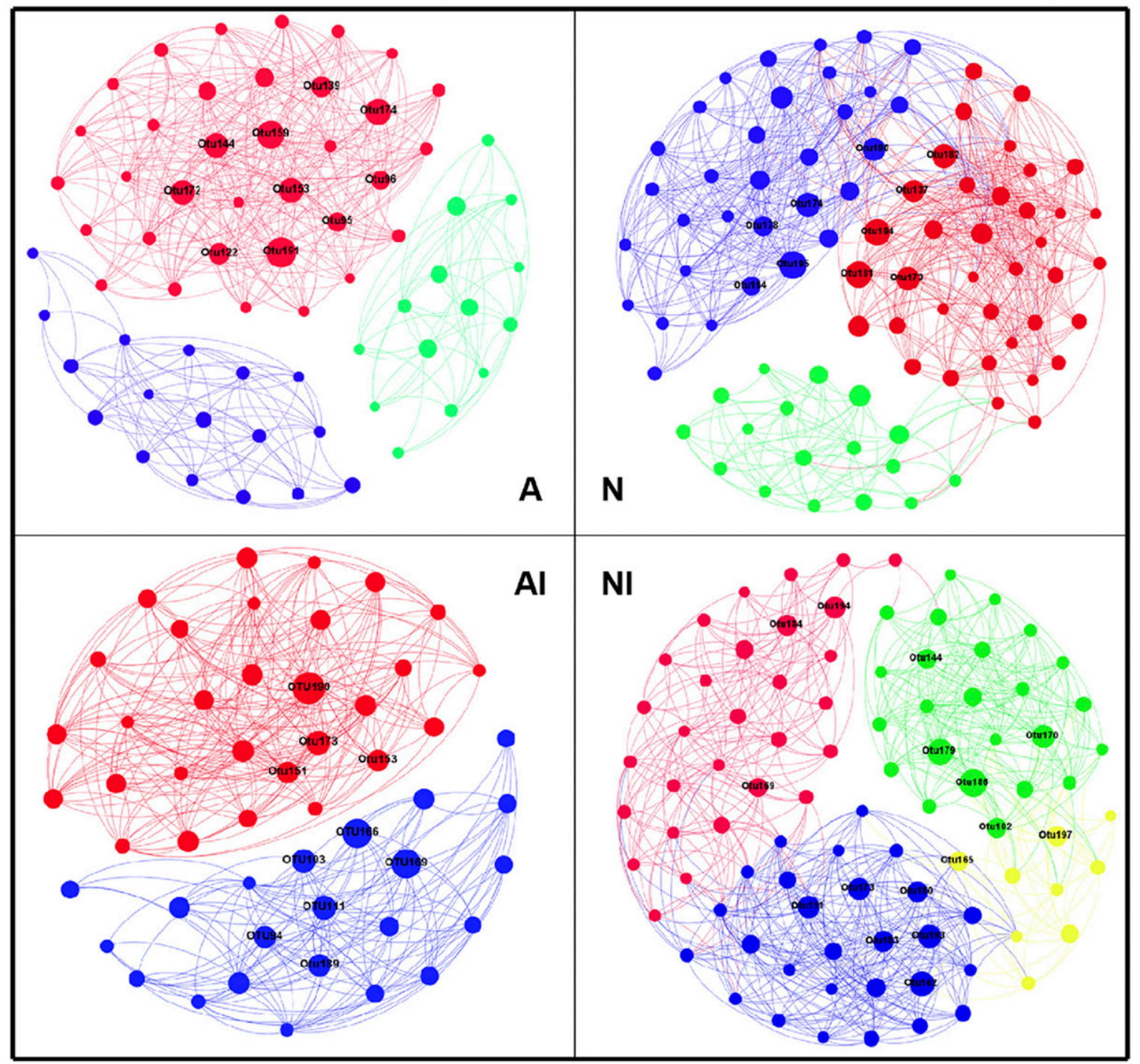

Fig. 5. The network analysis based on Pearson correlations. The different color nodes belong to different modules, the size of the nodes are arranged according to the degree of connection, and the hubs of each treatment were identified as the top 10 number of connections and marked by corresponding fungal genera code (number of operational taxonomic units). A = with ammonium fertilizer, $\mathrm{N}=$ with nitrate fertilizer, $\mathrm{AI}=$ with ammonium fertilizer and inoculated with $F$. oxysporum f. sp. cucumerinum, and $\mathrm{NI}=$ with nitrate fertilizer and inoculated with $F$. oxysporum f. sp. cucumerinum. 


\section{MATERIALS AND METHODS}

Pathogen incubation and spore suspension preparation.

Fusarium oxysporum f. sp. cucumerinum was provided by the Laboratory of Plant-Microbe Interactions at Nanjing Agricultural University, China. F. oxysporum f. sp. cucumerinum isolates were excised from the margins of potato dextrose agar plate cultures (as $1 \mathrm{~mm}^{2}$ blocks) and inoculated into $1 \mathrm{~L}$ Erlenmeyer flasks containing Bilay's medium for 7 days at $28^{\circ} \mathrm{C}$ (Tan et al. 2011). Conidia were then resuspended in sterile water and counted using a hemocytometer. The $F$. oxysporum $\mathrm{f}$. sp. cucumerinum spore suspension containing $10^{7}$ spores $\mathrm{ml}^{-1}$ was prepared for inoculation.

\section{Greenhouse experiment design.}

Pot experiments were conducted in a greenhouse under which the averaged month insolation incident, daylight hours,

Table 2. Correlations and topological properties of rhizosphere fungal networks in cucumber plants ${ }^{\mathrm{s}}$

\begin{tabular}{lcccc}
\hline Network properties & A & N & AI & NI \\
\hline Number of nodes $^{\mathrm{t}}$ & 65 & 86 & 51 & 89 \\
Number of edges $^{\mathrm{u}}$ & 396 & 610 & 345 & 621 \\
Modularity $^{\mathrm{v}}$ & 0.56 & 0.51 & 0.45 & 0.57 \\
Number of communities $^{\mathrm{w}}$ & 3 & 3 & 2 & 4 \\
Network diameter $^{\mathrm{x}}$ & 10 & 9 & 3 & 9 \\
Average path length $^{\mathrm{y}}$ & 1.83 & 1.36 & 2.42 & 1.69 \\
Average degree $^{\mathrm{z}}$ & 6.09 & 7.09 & 6.76 & 6.98 \\
\hline
\end{tabular}

$\mathrm{s} \mathrm{A}=$ with ammonium fertilizer, $\mathrm{N}=$ with nitrate fertilizer, $\mathrm{AI}=$ with ammonium fertilizer and inoculated with $F$. oxysporum $\mathrm{f}$. sp. cucumerinum, and $\mathrm{NI}=$ with nitrate fertilizer and inoculated with $F$. oxysporum $\mathrm{f}$. sp. cucumerinum.

t Microbial taxon (at genus level) with at least one significant $(P<0.01)$ and strong (Pearson correlation of 0.60 to 0.93 ).

u Number of connections or correlations obtained by Pearson correlation analysis.

$\checkmark$ The capability of the nodes to form highly connected communities, that is, a structure with high density of between nodes connections (inferred by Gephi). This value refers to the difference between smaller groups inside the community.

w A community is defined as a group of nodes densely connected internally (Gephi).

$\mathrm{x}$ The longest distance between nodes in the network, measured in number of edges (Gephi).

y Average network distance between all pairs of nodes or the average length off all edges in the network (Gephi).

$\mathrm{z}$ Average length between any two nodes or the average length off all edges in the network (Gephi). This value refers to the degree of association between all nodes in the network. air temperature, and relative humidity is $4.33 \mathrm{kWh} \mathrm{m}^{-2}$ day $^{-1}$, $13.06 \mathrm{~h}, 23.06^{\circ} \mathrm{C}$ and $77.66 \%$. Seeds of cucumber (Cucumis sativus L.), cultivar "Jinyan 4" (susceptible to F. oxysporum f. sp. cucumerinum) obtained from the Vegetable Research Institute of Tianjin, China, were germinated in sterile quartz sand and transplanted when the first leaves emerged. $\mathrm{Cu}$ cumber seedlings were then transplanted to pots containing $3 \mathrm{~kg}$ of field soil, the soil is collected in a cucumber planting farmland, and the soil type is clayey soil. Before transplanting, solid powdered fertilizers were mixed well with the soil. Four treatments were applied: A, applying ammonium fertilizer $\left[\left(\mathrm{NH}_{4}\right)_{2} \mathrm{SO}_{4}\right] ; \mathrm{N}$, applying nitrate fertilizer $[\mathrm{Ca}$ $\left(\mathrm{NO}_{3}\right)_{2}$ ], AI, applying ammonium fertilizer inoculated with F. oxysporum f. sp. cucumerinum; NI, applying nitrate fertilizer inoculated with $F$. oxysporum f. sp. cucumerinum. All treatments contained the same amounts of nitrogen (Nitrogen, $\left.0.24 \mathrm{~g} \mathrm{~kg}^{-1}\right)$; phosphorus $\left(\mathrm{P}_{2} \mathrm{O}_{5}, 0.07 \mathrm{~g} \mathrm{~kg}^{-1}\right)$ and potassium $\left(\mathrm{K}_{2} \mathrm{O}, 0.27 \mathrm{~g} \mathrm{~kg}^{-1}\right)$ throughout the whole season and six repetitions were set for each treatment, that means 24 pots ( 1 seed per pot) were conducted each time. Tap water was used to irrigate daily to ensure the water content of the soil. On the 20 th day after transplanting, $100 \mathrm{ml}$ of the $F$. oxysporum $\mathrm{f}$. sp. cucumerinum spore suspension described above was evenly added to the soil for AI and NI treatments, and the same amount of sterile water was added in $\mathrm{A}$ and $\mathrm{N}$ treatments. The pot experiment was repeated three times to ensure the reliability of plant growth and disease occurrence, and we collected the soil in the last repetition for analysis and DNA extraction.

The disease degree is divided into four grades: 0 means that no wilting in plant; 1 means that $0-25 \%$ of leaves are wilting in the plant; 2 means $25-50 \%$ of leaves are wilting in the plant; 3 means $50-75 \%$ of leaves are wilting in the plant; 4 means $75-100 \%$ of leaves are wilting in the plant. The disease index is calculated according to the following formula:

$$
\begin{aligned}
& \text { Disease index }= \\
& \frac{\sum(\text { Each grade number } * \text { Number of plants at each grade })}{4 * \text { Total number of plants }}
\end{aligned}
$$

Three plants for each treatment were selected to take photos and Infrared images of cucumber leaves were obtained using an infrared camera (SC620, FLIR Systems, Goleta, CA, U.S.A.) with a spectral sensitivity ranging from 7.5 to $13 \mathrm{~mm}$ and a spatial resolution of $0.65 \mathrm{mrad}$, Digital thermograms

Table 3. Pearson analysis for the relative abundance of each fungal genus belonging to Pathotroph-Saprotroph, Pathotroph-Saprotroph-Symbiotroph and

\begin{tabular}{|c|c|c|c|}
\hline Genus & Pearson correlation coefficient ${ }^{\mathrm{z}}$ & $P>t$ & FUNGuild classification \\
\hline Auriculibuller & 0.25 & 0.01 & Pathotroph-Saprotroph-Symbiotroph \\
\hline Botrytis & -0.24 & 0.02 & Pathotroph-Saprotroph \\
\hline Basidiobolus & -0.23 & 0.02 & Saprotroph \\
\hline Fusicolla & -0.28 & 0.01 & Saprotroph \\
\hline Geastrum & -0.27 & 0.01 & Saprotroph \\
\hline Harzia & -0.2 & 0.04 & Saprotroph \\
\hline Humicola & -0.27 & 0.01 & Saprotroph \\
\hline Hypochnicium & -0.26 & 0.01 & Saprotroph \\
\hline Apodus & -0.27 & 0.01 & Saprotroph \\
\hline Pacispora & -0.25 & 0.01 & Saprotroph \\
\hline Phialemonium & -0.23 & 0.03 & Saprotroph \\
\hline Pisolithus & 0.25 & 0.01 & Saprotroph \\
\hline Purpureocillium & -0.22 & 0.03 & Saprotroph \\
\hline Schizangiella & -0.24 & 0.02 & Saprotroph \\
\hline Stachybotrys & 0.21 & 0.04 & Saprotroph \\
\hline Lulwoana & 0.23 & 0.02 & Saprotroph \\
\hline
\end{tabular}
Saprotroph with the F. oxysporum abundance ${ }^{\mathrm{y}}$

y As determined by quantitative PCR. The fungal genera listed are all significantly related to Fusarium oxysporum $(P<0.05)$.

${ }^{\mathrm{z}}$ Pearson correlation coefficient greater than 0 indicates positive correlation, and less than 0 indicates negative correlation. 
were analyzed using Therma CAM Researcher Professional 2.9 software (FLIR Systems).

\section{Rhizosphere soil sample collection.}

On the 38th day after transplanting, disease symptoms were observed and the soil samples were collected for further analysis. The rhizosphere soil samples consisted of roots was shaken vigorously to separate soil that was not tightly adhering to the roots. Then, $5 \mathrm{~g}$ of plant roots with firmly adhering soil was resuspended in $30 \mathrm{ml}$ of distilled water and vortexed for $1 \mathrm{~min}$ at high speed. The soil in the distilled water represented the rhizosphere used in our analyses. After vacuum freeze drying, one part was stored at $4{ }^{\circ} \mathrm{C}$ and used in soil chemical and microbiological testing while the other part was stored at $-80^{\circ} \mathrm{C}$ for molecular analyses. The water content of soil was between $18-20 \%$ when the samples were collected.

\section{Determination of soil chemical and microbiological properties.}

The chemical properties of the soil were determined according to established methods (Shen et al. 2015a; Luo et al. 2018). Briefly, soil pH was determined with a soil-to-water ratio of 1: 2.5 by $\mathrm{pH}$ meter (FE28-Meter). Soil total carbon (TC) and total nitrogen were determined using Elemental Analyzer (Vario MAX; Elementar, Germany). Soil nitrate $\left(\mathrm{NO}_{3}{ }^{-}-\right.$ $\mathrm{N})$ and ammonium $\left(\mathrm{NH}_{4}{ }^{+}-\mathrm{N}\right)$ contents were extracted with $2 \mathrm{M}$ $\mathrm{KCl}$ (Li et al. 2014) and were quantified by Bran+Luebbe GmbH-AutoAnalyzer 3 (Norderstedt, Germany). Available phosphorus in the soil was extracted using $\mathrm{NaHCO}_{3}$ (Motavalli and Miles 2002) and then measured by the molybdenum-blue method. Available potassium in the soil was extracted with $\mathrm{NH}_{4} \mathrm{OAc}$ (Zhang et al. 2013) and determined using a flame photometer. MBC and MBN were estimated according to the chloroform-fumigation extraction method and were calculated based on the difference between soil extracted with $0.5 \mathrm{M}$ $\mathrm{K}_{2} \mathrm{SO}_{4}$ in fumigated and the nonfumigated samples (Gregorich et al. 1991). Organic carbon in the extracts was quantified using an automated TOC analyzer (LiquiTOC II, Elementar Analyzer system $\mathrm{GmbH}$, Hanau, Germany), and organic nitrogen was detected using the Kjeldahl method. Conversion factors of 0.45 $\left(\mathrm{KE}_{\mathrm{C}}\right)$ and $0.54\left(\mathrm{KE}_{\mathrm{N}}\right)$ were used to calculate average $\mathrm{MBC}$ and MBN values.

\section{DNA extraction and quantitative PCR analysis.}

Random four rhizosphere soil samples of each treatment were prepared for extraction of total DNA. Soil total genomic DNA was extracted from $0.25 \mathrm{~g}$ soil using the PowerSoil DNA Isolation Kit (MoBio Laboratories Inc., Carlsbad, CA, U.S.A.) and dissolved into $50 \mu \mathrm{L}$ sterile distilled water aliquots following the manufacturer's instructions. Extracted DNA concentration and quality were determined with a NanoDrop 2000 spectrophotometer (Thermo Scientific, Waltham, MA, U.S.A.).

The isolated DNA samples were then used as templates for PCRs using the $F$. oxysporum f. sp. cucumerinum-specific SCAR primers FOF1 and FOR1 (Kozich et al. 2013; Shen et al. 2015a) which target the rRNA internal transcribed spacer. Quantitative real-time PCR (RT-qPCR) was performed using a 7500 Real-Time PCR System (Applied Biosystems). Each reaction $(20 \mu \mathrm{L}$ total volume) consisted of $10 \mu \mathrm{L}$ of SYBR Green PCR Master Mix (TAKARA), $0.4 \mu \mathrm{L}$ of ROX dye $(50 \times), 0.4 \mu \mathrm{L}$ of each primer $(10 \mu \mathrm{M}), 2 \mu \mathrm{L}$ of template DNA and $6.8 \mu \mathrm{L}$ of sterile distilled water. The PCR program was carried out at $95^{\circ} \mathrm{C}$ for $10 \mathrm{~min}$, followed by 40 cycles of $95^{\circ} \mathrm{C}$ for $15 \mathrm{~s}$ and $60^{\circ} \mathrm{C}$ for $1 \mathrm{~min}$. To evaluate amplification specificity, melt curve analyses were performed at the end of the PCR runs. The standard curves were generated according to a previous report
(Takara Bio Inc., Kusatsu, Japan). Gene copy numbers of the target group for each reaction were calculated from the standard curves. Three replicates were used for each sample and the results were expressed as $1 \mathrm{~g}$ values (target copy number per gram of soil).

\section{MiSeq high-throughput sequencing analysis.}

After removing the adaptors and primer sequences, the raw sequences were assembled for each sample using QIIME (Edgar 2013). Split sequences for each sample were merged using FLASH V1.2.7 (Magoč and Salzberg 2011). The sequences retained for each sample were processed following the established UPARSE pipeline (Lin et al. 2012). In brief, sequences with a quality score lower than 25 or having a length shorter than $200 \mathrm{bp}$ were removed. After discarding the singletons, the remaining reads were assigned to OTUs based on a $97 \%$ similarity threshold. Then, the chimeric sequences were removed. Finally the fungal OTUs were classified using UNITE database (Kõljalg et al. 2013) using the naïve Bayesian classifier implemented in Mothur with an $80 \%$ confidence threshold (Schloss et al. 2009). The sequences were deposited in the NCBI Sequence Read Archive (SRA) database (BioProject: PRJNA531358; SRP: SRP191361).

\section{Statistical analysis.}

The Chao1 and Shannon indexes were calculated to estimate $\alpha$-diversity of each sample using mothur (Schloss et al. 2009). Network analysis was carried with the interactive platform Gephi with Fruchterman-Reingold layout (Bastian and Heymann 2009) from which number of nodes, number of edges, modularity, number of communities, network diameter, average path length and average degree were calculated (Newman 2006). All possible Pearson's rank correlation coefficients were calculated

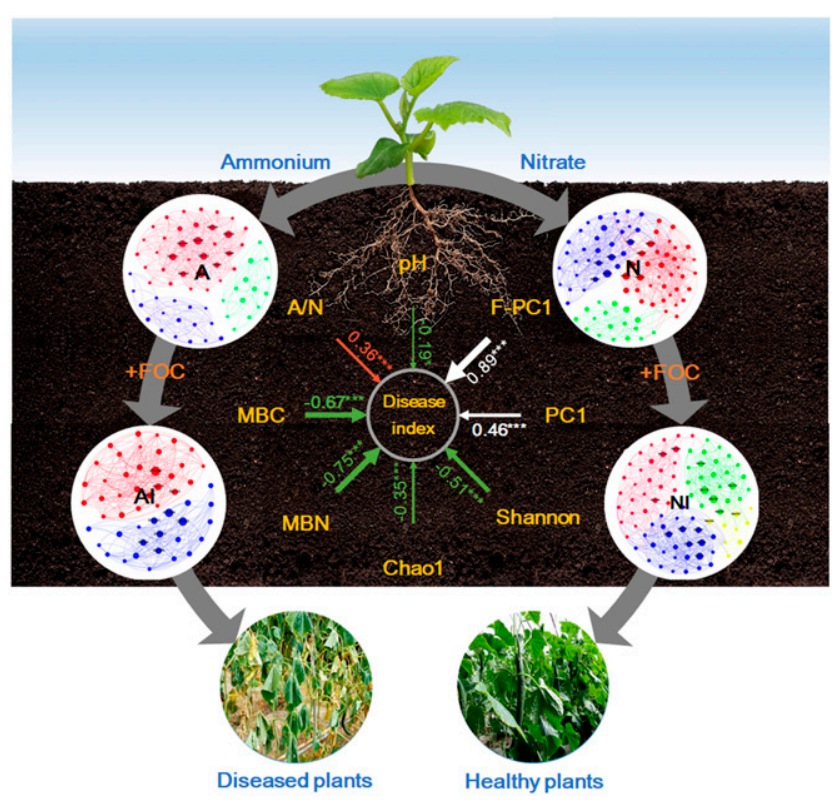

Fig. 6. The possible resistant mechanism of nitrate to cucumber Fusarium wilt. $\mathrm{MBC}=$ soil microbial biomass carbon and $\mathrm{MBN}=$ soil microbial biomass nitrogen. Alpha-diversity calculations include Chao1 and Shannon indices, PC1 (the first principal component (explained 47\%) of the principal component analyses (PCAs) based on fungal community), F-PC1 (the first principal component (explained 72\%) of the PCAs based on PathotrophSaprotroph, Pathotroph-Saprotroph-Symbiotroph, and Saprotroph abundance). Red arrows indicate negative correlation, green arrows indicate positive correlation, and the white arrows mean the correlation analysis only indicates correlation strength, with the correlation coefficient analysis shown by the arrows. 
via mothur to analyze the networks. The correlation data were filtered with a cut-off at an absolute r-value of 0.60 to 0.93 (Zhu et al. 2016), and a significant $P$ value of $<0.01$ to improve the accuracy of the networks. For constrained PCoA analysis, anova.cca from the vegan package in $\mathrm{R}$ was employed. Permutational analysis of variance was employed for permutation testing with the number of permutations set at 999. In PCA, the first principal component was linked to community variation (PC1) and community functional variation (F-PC1) of the rhizosphere fungi.

To determine statistical differences between the treatments, the Statistical Analysis of Metagenomic Profiles (STAMP) software package was used (Parks and Beiko 2010), corrected $P$ values were calculated using Welch's $t$ test (Newcombe 1998) and Benjamini-Hochberg false discovery rate approach (Benjamini and Hochberg 1995). Significant effects of fungal genus as classified by FUNGuild on rhizospheric $F$. oxysporum f. sp. cucumerinum were also tested using Pearson's correlation (Kumar et al. 2013; Nguyen et al. 2016).

Data were compared using one-way analysis of variance at the end of each bioassay in the IBM SPSS 19.0 software program (SPSS Inc.). We used general linear models (GLM) with "block" as a random effect and "treatments" as fixed effects. The effect of fungal function classification on rhizosphere F. oxysporum f. sp. cucumerinum was also tested with GLM.

\section{LITERATURE CITED}

Amtmann, A., Troufflard, S., and Armengaud, P. 2008. The effect of potassium nutrition on pest and disease resistance in plants. Physiol. Plant. 133:682-691.

Bastian, M., and Heymann, S. 2009. Gephi: An open source software for exploring and manipulating networks. Proceedings of the Third International ICWSM Conference, May 2009. AAAI, Palo Alto, CA, U.S.A.

Benjamini, Y., and Hochberg, Y. 1995. Controlling the false discovery rate: A practical and powerful approach to multiple testing. J. R. Stat. Soc. 57: 289-300.

Bödeker, I. T. M., Lindahl, B. D., Olson, Å., Clemmensen, K. E., and Treseder, K. 2016. Mycorrhizal and saprotrophic fungal guilds compete for the same organic substrates but affect decomposition differently. Funct. Ecol. 30:1967-1978.

Celar, F. 2003. Competition for ammonium and nitrate forms of nitrogen between some phytopathogenic and antagonistic soil fungi. Biol. Control 28:19-24.

Chapelle, E., Mendes, R., Bakker, P. A., and Raaijmakers, J. M. 2016. Fungal invasion of the rhizosphere microbiome. ISME J. 10:265-268.

Dordas, C. 2008. Role of nutrients in controlling plant diseases in sustainable agriculture. A review. Agron. Sustain. Dev. 28:33-46.

Durner, J., and Klessig, D. F. 1999. Nitric oxide as a signal in plants. Curr. Opin. Plant Biol. 2:369-374.

Edgar, R. C. 2013. UPARSE: Highly accurate OTU sequences from microbial amplicon reads. Nat. Methods 10:996-998.

Faust, K., and Raes, J. 2012. Microbial interactions: From networks to models. Nat. Rev. Microbiol. 10:538-550.

Gregorich, E. G., Voroney, R. P., and Kachanoski, R. G. 1991. Turnover of carbon through the microbial biomass in soils with different textures. Soil Biol. Biochem. 23:799-805.

Guo, J., Liu, W., Zhu, C., Luo, G., Kong, Y., Ling, N., Wang, M., Dai, J., Shen, Q., and Guo, S. 2017. Bacterial rather than fungal community composition is associated with microbial activities and nutrient-use efficiencies in a paddy soil with short-term organic amendments. Plant Soil 424:335-349.

Gupta, K. J., Brotman, Y., Segu, S., Zeier, T., Zeier, J., Persijn, S. T., Cristescu, S. M., Harren, F. J., Bauwe, H., Fernie, A. R., Kaiser, W. M., and Mur, L. A. 2013. The form of nitrogen nutrition affects resistance against Pseudomonas syringae pv. phaseolicola in tobacco. J. Exp. Bot. 64:553-568

Haas, D., and Défago, G. 2005. Biological control of soil-borne pathogens by fluorescent pseudomonads. Nat. Rev. Microbiol. 3:307-319.

Hakulinen, N., Turunen, O., Jänis, J., Leisola, M., and Rouvinen, J. 2003 Three-dimensional structures of thermophilic beta-1,4-xylanases from Chaetomium thermophilum and Nonomuraea flexuosa. Comparison of twelve xylanases in relation to their thermal stability. Eur. J. Biochem. 270:1399-1412.

Hartmann, A., Schmid, M., Tuinen, D., and Berg, G. 2008. Plant-driven selection of microbes. Plant Soil 321:235-257.

Heuck, C., Weig, A., and Spohn, M. 2015. Soil microbial biomass C:N:P stoichiometry and microbial use of organic phosphorus. Soil Biol. Biochem. 85:119-129.

Hoffland, E., Jeger, M. J., and van Beusichem, M. L. 2000. Effect of nitrogen supply rate on disease resistance in tomato depends on the pathogen. Plant Soil 218:239-247.

Hortal, S., Bastida, F., Armas, C., Lozano, Y. M., Moreno, J. L., García, C., and Pugnaire, F. I. 2013. Soil microbial community under a nurse-plant species changes in composition, biomass and activity as the nurse grows. Soil Biol. Biochem. 64:139-146.

Hu, J., Wei, Z., Friman, V. P., Gu, S. H., Wang, X. F., Eisenhauer, N., Yang, T. J., Ma, J., Shen, Q. R., Xu, Y. C., and Jousset, A. 2016. Probiotic diversity enhances rhizosphere microbiome function and plant disease suppression. MBio 7:e01790-16.

Huang, Y., Xiao, X., Huang, H., Jing, J., Zhao, H., Wang, L., and Long, X. E. 2018. Contrasting beneficial and pathogenic microbial communities across consecutive cropping fields of greenhouse strawberry. Appl Microbiol. Biot. 102:5717-5729.

Huber, D. M., and Watson, R. D. 1974. Nitrogen form and plant disease Annu. Rev. Phytopathol. 12:139-165.

Hwang, S.-C., and Ko, W.-H. 2004. Cavendish banana cultivars resistant to Fusarium wilt acquired through somaclonal variation in Taiwan. Plant Dis. 88:580-588.

Jousset, A., Becker, J., Chatterjee, S., Karlovsky, P., Scheu, S., and Eisenhauer, N. 2014. Biodiversity and species identity shape the antifungal activity of bacterial communities. Ecology 95:1184-1190.

Klein, E., Katan, J., and Gamliel, A. 2016. Soil suppressiveness by organic amendment to Fusarium disease in cucumber: Effect on pathogen and host. Phytoparasitica 44:239-249.

Kõljalg, U., Nilsson, R. H., Abarenkov, K., Tedersoo, L., Taylor, A. F. S., Bahram, M., Bates, S. T., Bruns, T. D., Bengtsson-Palme, J., Callaghan, T. M., Douglas, B., Drenkhan, T., Eberhardt, U., Dueñas, M., Grebenc, T., Griffith, G. W., Hartmann, M., Kirk, P. M., Kohout, P., Larsson, E., Lindahl, B. D., Lücking, R., Martín, M. P., Matheny, P. B., Nguyen, N. H., Niskanen, T., Oja, J., Peay, K. G., Peintner, U., Peterson, M., Põldmaa, K., Saag, L., Saar, I., Schüßler, A., Scott, J. A., Senés, C., Smith, M. E., Suija, A., Taylor, D. L., Telleria, M. T., Weiss, M., and Larsson, K. H. 2013. Towards a unified paradigm for sequence-based identification of fungi. Mol. Ecol. 22:5271-5277.

Kozich, J. J., Westcott, S. L., Baxter, N. T., Highlander, S. K., and Schloss, P. D. 2013. Development of a dual-index sequencing strategy and curation pipeline for analyzing amplicon sequence data on the MiSeq Illumina sequencing platform. Appl. Environ. Microbiol. 79:5112-5120.

Kumar, M., Yadav, A. N., Tiwari, R., Prasanna, R., and Saxena, A. K. 2013. Deciphering the diversity of culturable thermotolerant bacteria from Manikaran hot springs. Ann. Microbiol. 64:741-751.

Li, M., Zhou, X., Zhang, Q., and Cheng, X. 2014. Consequences of afforestation for soil nitrogen dynamics in central China. Agric. Ecosyst. Environ. 183:40-46.

Lin, X., Feng, Y., Zhang, H., Chen, R., Wang, J., Zhang, J., and Chu, H 2012. Long-term balanced fertilization decreases arbuscular mycorrhizal fungal diversity in an arable soil in North China revealed by 454 pyrosequencing. Environ. Sci. Technol. 46:5764-5771.

Liu, B., Gumpertz, M. L., Hu, S., and Ristaino, J. B. 2007a. Long-term effects of organic and synthetic soil fertility amendments on soil microbial communities and the development of southern blight. Soil Biol. Biochem. 39:2302-2316.

Liu, B., Tu, C., Hu, S., Gumpertz, M., and Ristaino, J. B. 2007b. Effect of organic, sustainable, and conventional management strategies in grower fields on soil physical, chemical, and biological factors and the incidence of Southern blight. Appl. Soil Ecol. 37:202-214.

Long, D. H., Lee, F. N., and TeBeest, D. O. 2000. Effect of nitrogen fertilization on disease progress of rice blast on susceptible and resistan cultivars. Plant Dis. 84:403-409.

López-Berges, M. S., Rispail, N., Prados-Rosales, R. C., and Di Pietro, A. 2010. A nitrogen response pathway regulates virulence functions in Fusarium oxysporum via the protein kinase TOR and the bZIP protein MeaB. Plant Cell 22:2459-2475.

Luo, G. W., Rensing, C., Chen, H., Liu, M. Q., Wang, M., Guo, S. W., Ling, N., and Shen, Q. R. 2018. Deciphering the associations between soil microbial diversity and ecosystem multifunctionality driven by longterm fertilization management. Funct. Ecol. 32:1103-1116.

Magoč, T., and Salzberg, S. L. 2011. FLASH: Fast length adjustment of short reads to improve genome assemblies. Bioinformatics 27:2957-2963. 
Mallon, C. A., Le Roux, X., van Doorn, G. S., Dini-Andreote, F., Poly, F., and Salles, J. F. 2018. The impact of failure: Unsuccessful bacterial invasions steer the soil microbial community away from the invader's niche. ISME J. 12:728-741.

Mendes, L. W., Mendes, R., Raaijmakers, J. M., and Tsai, S. M. 2018a. Breeding for soil-borne pathogen resistance impacts active rhizosphere microbiome of common bean. ISME J. 12:3038-3042.

Mendes, L. W., Raaijmakers, J. M., de Hollander, M., Mendes, R., and Tsai, S. M. 2018b. Influence of resistance breeding in common bean on rhizosphere microbiome composition and function. ISME J. 12:212-224.

Meng, T., Yang, Y., Cai, Z., and Ma, Y. 2017. The control of Fusarium oxysporum in soil treated with organic material under anaerobic condition is affected by liming and sulfate content. Biol. Fertil. Soils 54:295-307.

Motavalli, P., and Miles, R. 2002. Soil phosphorus fractions after 111 years of animal manure and fertilizer applications. Biol. Fertil. Soils 36:35-42.

Mur, L. A. J., Simpson, C., Kumari, A., Gupta, A. K., and Gupta, K. J. 2017. Moving nitrogen to the centre of plant defence against pathogens. Ann. Bot. 119:703-709.

Nel, B., Steinberg, C., Labuschagne, N., and Viljoen, A. 2007. Evaluation of fungicides and sterilants for potential application in the management of Fusarium wilt of banana. Crop Prot. 26:697-705.

Newcombe, R. G. 1998. Two-sided confidence intervals for the single proportion: Comparison of seven methods. Stat. Med. 17:857-872.

Newman, M. E. 2006. Modularity and community structure in networks. Proc. Natl. Acad. Sci. U.S.A. 103:8577-8582.

Nguyen, N. H., Song, Z., Bates, S. T., Branco, S., Tedersoo, L., Menke, J., Schilling, J. S., and Kennedy, P. G. 2016. FUNGuild: An open annotation tool for parsing fungal community datasets by ecological guild. Fungal Ecol. 20:241-248.

Parks, D. H., and Beiko, R. G. 2010. Identifying biologically relevant differences between metagenomic communities. Bioinformatics 26: 715-721.

Poudel, R., Jumpponen, A., Schlatter, D. C., Paulitz, T. C., Gardener, B. B., Kinkel, L. L., and Garrett, K. A. 2016. Microbiome networks: A systems framework for identifying candidate microbial assemblages for disease management. Phytopathology 106:1083-1096.

Rosenzweig, N., Tiedje, J. M., Quensen, J. F., 3rd, Meng, Q., and Hao, J. J. 2012. Microbial communities associated with potato common scab-suppressive soil determined by pyrosequencing analyses. Plant Dis. 96:718-725.

Sasse, J., Martinoia, E., and Northen, T. 2018. Feed your friends: Do plant exudates shape the root microbiome? Trends Plant Sci. 23:25-41.

Schloss, P. D., Westcott, S. L., Ryabin, T., Hall, J. R., Hartmann, M., Hollister, E. B., Lesniewski, R. A., Oakley, B. B., Parks, D. H., Robinson, C. J., Sahl, J. W., Stres, B., Thallinger, G. G., Van Horn, D. J., and Weber, C. F. 2009. Introducing mothur: Open-source, platform-independent, community-supported software for describing and comparing microbial communities. Appl. Environ. Microbiol. 75:7537-7541.

Shen, Z., Ruan, Y., Wang, B., Zhong, S., Su, L., Li, R., and Shen, Q. 2015a. Effect of biofertilizer for suppressing Fusarium wilt disease of banana as well as enhancing microbial and chemical properties of soil under greenhouse trial. Appl. Soil Ecol. 93:111-119.

Shen, Z. Z., Ruan, Y. Z., Xue, C., Zhong, S. T., Li, R., and Shen, Q. R. 2015b. Soils naturally suppressive to banana Fusarium wilt disease harbor unique bacterial communities. Plant Soil 393:21-33.
Soustrade, I., Gagnevin, L., Roumagnac, P., Gambin, O., Guillaumin, D., and Jeuffrault, E. 2000. First report of anthurium blight caused by Xanthomonas axonopodis pv. dieffenbachiae in Reunion Island. Plant Dis. 84:1343.

Tan, D. C., Flematti, G. R., Ghisalberti, E. L., Sivasithamparam, K., Chakraborty, S., Obanor, F., and Barbetti, M. J. 2011. Mycotoxins produced by Fusarium species associated with annual legume pastures and 'sheep feed refusal disorders' in Western Australia. Mycotoxin Res. 27:123-135.

Tiburcio, A. F., Altabella, T., Bitrián, M., and Alcázar, R. 2014. The roles of polyamines during the lifespan of plants: From development to stress. Planta 240:1-18.

van Bruggen, A. H., Gamliel, A., and Finckh, M. R. 2016. Plant disease management in organic farming systems. Pest Manag. Sci. 72: 30-44.

van Elsas, J. D., Chiurazzi, M., Mallon, C. A., Elhottova, D., Kristufek, V., and Salles, J. F. 2012. Microbial diversity determines the invasion of soil by a bacterial pathogen. Proc. Natl. Acad. Sci. U.S.A. 109: 1159-1164.

Walters, D., Cowley, T., and Mitchell, A. 2002. Methyl jasmonate alters polyamine metabolism and induces systemic protection against powdery mildew infection in barley seedlings. J. Exp. Bot. 53:747-756.

Wang, B., Li, R., Ruan, Y., Ou, Y., Zhao, Y., and Shen, Q. 2015. Pineapple-banana rotation reduced the amount of Fusarium oxysporum more than maize-banana rotation mainly through modulating fungal communities. Soil Biol. Biochem. 86:77-86.

Wang, M., Sun, Y., Gu, Z., Wang, R., Sun, G., Zhu, C., Guo, S., and Shen, Q. 2016. Nitrate protects cucumber plants against Fusarium oxysporum by regulating citrate exudation. Plant Cell Physiol. 57:2001-2012.

Wei, Z., Yang, T., Friman, V. P., Xu, Y., Shen, Q., and Jousset, A. 2015 Trophic network architecture of root-associated bacterial communities determines pathogen invasion and plant health. Nat. Commun. 6:8413.

Xiao, D., Xiao, S., Ye, Y., Zhang, W., He, X., and Wang, K. 2018. Microbial biomass, metabolic functional diversity, and activity are affected differently by tillage disturbance and maize planting in a typical karst calcareous soil. J. Soils Sediments 19:809-821.

Xiong, W., Li, R., Ren, Y., Liu, C., Zhao, Q., Wu, H., Jousset, A., and Shen, Q. 2017. Distinct roles for soil fungal and bacterial communities associated with the suppression of vanilla Fusarium wilt disease. Soil Biol. Biochem. 107:198-207.

Xiong, W., Zhao, Q., Xue, C., Xun, W., Zhao, J., Wu, H., Li, R., and Shen, Q. 2016. Comparison of fungal community in black pepper-vanilla and vanilla monoculture systems associated with vanilla Fusarium wilt disease. Front. Microbiol. 7:117.

Zhang, S. L., Zhang, X. Y., Liu, X. B., Liu, W., and Liu, Z. H. 2013. Spatial distribution of soil nutrient at depth in black soil of Northeast China: A case study of soil available potassium. Nutr. Cycl. Agroecosyst. 95: 319-331.

Zhou, J., Wang, M., Sun, Y., Gu, Z., Wang, R., Saydin, A., Shen, Q., and Guo, S. 2017. Nitrate increased cucumber tolerance to Fusarium wilt by regulating fungal toxin production and distribution. Toxins (Basel) 9: 100.

Zhu, C., Ling, N., Guo, J., Wang, M., Guo, S., and Shen, Q. 2016. Impacts of fertilization regimes on arbuscular mycorrhizal fungal (AMF) community composition were correlated with organic matter composition in maize rhizosphere soil. Front. Microbiol. 7:1840. 